\title{
A propos du fax en cabinet médical
}

\section{Eberhard Wolff}

PD Dr rer. soc., rédacteur culture, histoire, société

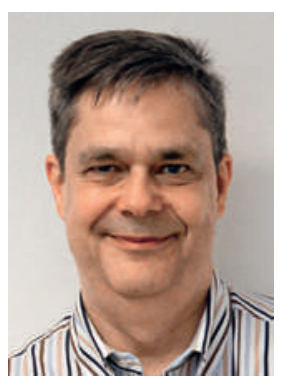

Récemment, il m’a fallu envoyer rapidement un document à un monsieur vivant dans une résidence pour personnes âgées. Impossible par Internet. La voie postale était trop lente. Le monsieur m'a dit que je n'avais qu'à lui faxer.

Un fax?!?! Cette «technologie préhistorique» a disparu depuis près de dix ans de mon univers privé et professionnel. Même la résidence pour personnes âgées (!) n'indiquait aucun numéro de fax sur Internet.

Mais n'enterrons pas le fax trop vite [1]. Même, voire surtout, dans les cabinets médicaux. L'étude SISA II [2] nous montre que faxer un document reste un geste naturel [3] dans la plupart des cabinets médicaux suisses, même quand ils sont informatisés. $69 \%$ des validations finales des laboratoires "medica" arrivent par exemple aussi (voire seulement) par fax dans les cabinets. Les défenseurs de la cybersanté y voient une carence et veulent changer cela. Le cabinet médical totalement numérisé, en réseau, menace à l'horizon. Je voudrais inverser la problématique: pourquoi les fax clignotent-ils (encore) dans les cabinets médicaux? C'est simple: quand les résultats du petit laboratoire arrivent ou que l'ordonnance urgente doit être envoyée à la petite pharmacie, et qu'il n'y a pas d'interface numérique, même le plus moderne des cabinets médicaux doit communiquer par fax. Mais cela va devenir de plus en plus rare.

Quelques cabinets veulent régler leurs affaires par fax, par habitude. Ou ils n'ont confiance qu'en cet appareil pour que les informations parviennent au destinataire prévu et pas à un autre. Le fax est devenu pour eux le «moteur Diesel» de la communication du cabinet.

Mais le fax matériel peut être pratique dans certaines situations. J'ai entendu parler de plus d'un cabinet pour lesquels les fax papier des résultats de laboratoire suivaient tout un parcours organisé. Déjà, en arrivant, le papier attire l'attention l'attention (parfois, seules les valeurs pathologiques sont faxées). Il est facile à garder en vue. Suivant le degré d'urgence, il est destiné à différents dossiers et collaborateurs. On conserve un aperçu des affaires en cours, le canal média reste contrôlable et ne se ramifie pas. On peut emporter le fax n'importe où, pas besoin de redémarrer quoique ce soit sur l'ordinateur le plus proche. Et ce n'est pas un problème si on renverse du café dessus. En ce sens, le fax garantit la qualité du cabinet, résultat du sens pratique sur place.
Manifestement, le secteur du bâtiment apprécie aussi le fax. Peut-être qu'il est plus vivant dans les domaines où on travaille physiquement sur l'homme ou les matériaux, et dans de nombreux endroits.

A vrai dire, même pour moi il serait plus simple de glisser dans le fax les corrections manuscrites de mes articles du BMS plutôt que de les envoyer par e-mail à Muttenz. Certains procèdent (encore) ainsi, déclare notre directrice de rédaction, Tanja Kühnle, me transformant malgré moi en néo-faxeur.

Et vous? Si vous tenez entre les mains une version imprimée de cet article: percevez-vous le doux plaisir de toucher et de tourner les pages, l'odeur de l'encre fraîche, les légers reflets du papier couché?

Deux fax de résultats des laboratoires «medica» sur trois arrivent aussi, de façon tout à fait ingénieuse, par voie électronique. Je pense qu'un cabinet médical ordinaire travaille constamment avec au moins cinq moyens de communication. Les chercheurs en cybersanté appellent cela la communication patchwork [2]. Ils estiment que c'est inefficace et qu'il faut y remédier. L'idée d'un «cabinet sans papier» est une sorte de leitmotiv: quand les ordinateurs sont apparus, on a rêvé de «bureaux sans papier». Nous savons ce qu'il en est advenu.

Mais la face cachée de la cybersanté, l'ordinaire, c'est la communication "patchwork», dans le cabinet médical comme dans le reste de la vie. Le chiffrage du gain de productivité du flux de données électronique n'est pas le seul aspect de la qualité de communication d'un cabinet. Petit quiz: quels moyens ont proposé les services de la FMH l'an dernier pour s'inscrire à l'atelier consacré à l'informatique au cabinet médical, dans le BMS? La réponse est: page d'accueil (unique accès au formulaire Internet), fax et téléphone, tous à pied d'égalité. Cela ne me semble pas incohérent, mais plutôt sympathique et réaliste, car cette communication patchwork est acceptée comme une normalité.

Sans compter que j'ai finalement pu envoyer mon fax à la résidence pour personnes âgées, en passant par le service fax sur Internet. L'établissement avait en effet encore sous la main un appareil qui fonctionnait. J'ai dégoté le numéro sur une vieille page Internet non effacée. Je fais le pari qu'un certain patchwork ne disparaîtra jamais. Secrètement, à défaut de persister publiquement. 